\title{
Gestión de la seguridad y la salud en el trabajo durante la construcción de obras de infraestructura vial en los departamentos de Atlántico, Magdalena y Bolívar*
}

\section{Occupational safety and health management system during the construction of road infrastructure in the departments of Atlántico, Magdalena and Bolivar}

Tania Paola Libonatti Madrid**

Recibido: 20 de octubre de 2013

Revisado: 22 de marzo de 2014

Aceptado: 27 de junio de 2014

Convenio USTA - ICONTEC

\section{RESUMEN}

Esta investigación describe la gestión en seguridad y salud en el trabajo (SST) que han llevado a cabo las empresas de construcción de obras de infraestructura vial en los departamentos de Atlántico, Magdalena y Bolívar, para incidir sobre su tasa de accidentalidad. Luego de una revisión de la literatura se definen las variables e hipótesis de trabajo, posteriormente se diseña un cuestionario como instrumento de recolección de datos que es validado por un sistema de jueces expertos. Se realiza un análisis de correlación para determinar la relación entre la existencia de un sistema de gestión de seguridad y salud en el trabajo (SG-SST), el cumplimiento de la legislación y el nivel de accidentalidad en las obras viales.

Los resultados permiten concluir que no hay incidencia del SG-SST sobre el nivel de accidentalidad. Además, se puede afirmar que hay relación entre el cumplimiento de la legislación y el nivel de accidentalidad.

Palabras clave: construcción de carreteras, tasa de accidentalidad, sistema de gestión de la seguridad y salud en el trabajo.

\footnotetext{
* Trabajo de grado para optar al título de Magister en Calidad y Gestión Integral dirigido por Mg. Orlando Mauricio Rodríguez Castaño.

** Tania Paola Libonatti Madrid, Ingeniero Industrial, Magíster en Calidad y Gestión Integral, Correo Electrónico: tanialibonatti@hotmail.com
} 


\section{ABSTRACT}

This research describes the management of occupational safety and health (OSH) that have been implemented by road infrastructure companies within the departments of Atlántico, Magdalena and Bolivar, to influence their accident rate. After a literature review variables and working hypotheses are defined, then a questionnaire is designed as a data collection instrument that is validated by a system of expert judges. A correlation analysis is conducted to determine the relationship between the existence of a safety management system and occupational health (OSH-MS), the compliance with the legislation and the level of accidents in road works. Results show that there is no incidence of OS-HMS on the accidents rate. But we can say that if there is relationship between law enforcement and the accidents rate.

Keywords: Road construction, accident rate, occupational health and safety management system.

\section{INTRODUCCIÓN}

El sector de la construcción juega un papel significativo en la economía del país, "ya que representa el $6.7 \%$ del PIB y el $5.1 \%$ del empleo total"1. En Colombia el sector de la construcción de obras de infraestructura vial es importante para la economía, el desarrollo y el crecimiento del país. Así mismo, los trabajadores de obras de infraestructura vial son de vital importancia para mantener y mejorar el nivel de seguridad y confort para los usuarios de la vía.

1 FEDERACIÓN INTERAMERICANA DE LA INDUSTRIA DE LA CONSTRucción. Evolución de la Economía de los Países Miembros de la FIIC 2009-2010. México, D.F. Octubre, 2010. [citado en 2011-07-12]. 42 p. Disponible en Internet: http://www.fiic. la/documentos/Evolucion\%20Economia\%20Paises\%20FIIC\%20 2009-2010.pdf
Los accidentes de trabajo en obras viales se deben a factores tales como las condiciones no favorables de iluminación, temperaturas extremas, el flujo de vehículos, la falta de pericia de los conductores y la falta de control del tráfico².

También se presentan enfermedades laborales características del sector de la construcción vial, por ejemplo, el Center for Construction Research and Training $(\mathrm{CPWR})^{3}$ expone que los trabajadores de las carreteras están propensos a adquirir asma por inhalaciones de sustancias y materiales utilizados en las obras. Según la Federación de Aseguradores Colombianos (FASECOLDA) ${ }^{4}$ en Colombia el sector de la construcción se caracteriza por el incremento ascendente de las estadísticas de accidentes de trabajo y enfermedades laborales a lo largo de los últimos años

Aunque con los años se han realizado grandes avances en la mejora de las normas de seguridad y salud en el trabajo, el número de accidentes de trabajo y enfermedades laborales sigue siendo elevado. El presente estudio describe cuál es la gestión en seguridad y salud

2 LI, Yingfeng, BAI, Yong. Comparison of characteristics between fatal and injury accidents in the highway construction zones. Safety Science [online]. 2008, vol. 46. [cited on 2011-05-03]. 657 p. Available from Internet: http://www.workzonesafety. org/files/documents/database_documents/Publication8794. pdf. ISSN 0925-7535.

3 THE CENTER FOR CONSTRUCTION RESEARCH AND TRAINING. Asthma in Heavy and Highway Construction Workers Exposed to Silica [online]. OLIVER, L. Christine, MIRACLEMCMAHILL, Heidi. Brookline, Massachusetts. July 2003. [cited on 2011-05-03]. p.4-5. Available from Internet: http://www.cpwr.com/ pdfs/pubs/research_pubs/oliver_Asthma.pdf

4 FEDERACIÓN DE ASEGURADORES COLOMBIANOS. Indicadores Técnicos: Encuesta Sistema de Información Gremial. Sistema de Información Estadística [en línea]. Bogotá, D.C. 2011. [citado en 2011-11-25]. Disponible en Internet: http://www.fasecolda.com/ fasecolda/BancoConocimiento/R/riesgos_profesionales_estadisticas_del_ramofinal_indicadores_tecnicos/riesgos_profesionales_estadisticas_del_ramofinal___indicadores_tecnicos.asp 
en el trabajo que realizan las empresas de construcción de obras viales, y cuál es su incidencia sobre la tasa de accidentalidad. Para ello se realiza una revisión de la literatura sobre el tema y posteriormente se evalúa el grado de cumplimiento de la legislación y la normatividad nacional sobre la gestión de la seguridad y salud en el trabajo, por parte de las empresas del sector.

Posteriormente se elabora y aplica una propuesta metodológica para la autoevaluación de la gestión en seguridad y salud en el trabajo en las empresas dedicadas a la construcción de obras de infraestructura vial y se realiza una prueba de correlación.

\section{METODOLOGÍA}

En la metodología de la investigación científica el concepto de técnicas de recolección de información alude a los procedimientos mediante los cuales se generan informaciones válidas y confiables ${ }^{5}$. La población del estudio constituye el $100 \%$ de las empresas que ejecutan obras de construcción y mantenimiento vial en las carreteras primarias concesionadas que comunican los departamentos del Atlántico, Bolívar y Magdalena. El periodo de estudio va de los años 2011 - 2013, y el número de empresas evaluadas son en total diecisiete.

El procedimiento implica la visita a dichas empresas aplicando como instrumento de recolección de datos un cuestionario de administración indirecta que "proviene del contexto de la investigación cuantitativa", aplicado por el investigador. A partir

5 YUNI, José y URBANO, Ariel. Técnicas para investigar II: recursos metodológicos para la preparación de proyectos de investigación. $2^{\circ}$ ed. Córdoba (Argentina): Brujas, 2006. Vol 02. p. 15-29.. ISBN 987-591-020-1.

6 Ibid. 63 p. de los datos recolectados, y con el ánimo de responder al problema de investigación, se definen las variables a estudiar en un cuadro de operacionalización de las variables, así como las hipótesis de trabajo. Luego se miden las variables definidas, y mediante el uso del estadístico chi cuadrado, se realiza la correlación. Más tarde se analizan los datos y se presentan los resultados del estudio.

\section{RESULTADOS}

A continuación se presentan los resultados más relevantes obtenidos a partir de encuestas, junto con un análisis de frecuencia de acuerdo con los rangos establecidos para cada variable del estudio. El $18 \%$ de las empresas encuestadas ha implementado un sistema de gestión de seguridad y salud en el trabajo (SG-SST), mientras que el $82 \%$ de estas no cuenta con un SG-SST.

Figura 1. Empresas que han implementado un SG-SST.

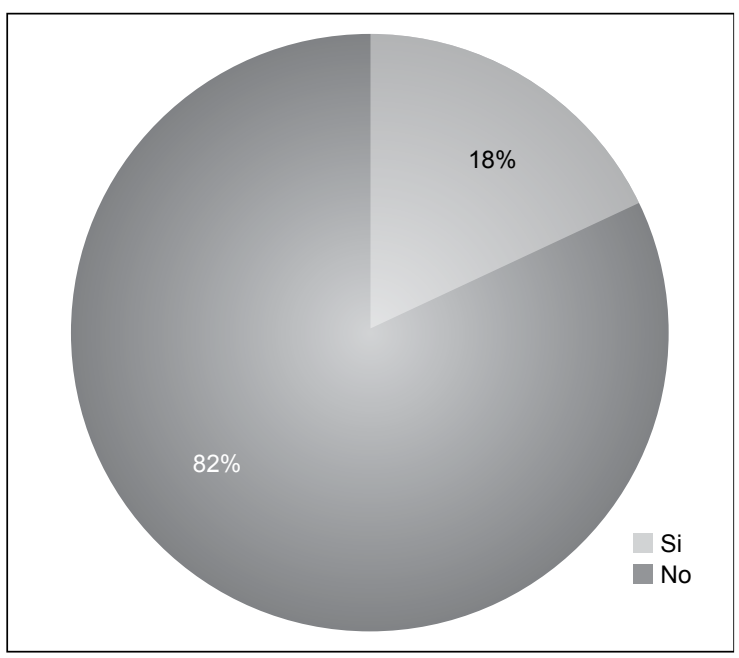

Fuente: elaboración propia.

El $94 \%$ de las empresas encuestadas tienen afiliados a sus trabajadores en el sistema general de riesgos laborales. El $6 \%$ restante cuenta con una póliza de seguro de vida. 
Figura 2. Empresas que cotizan en el sistema general de riesgos laborales.

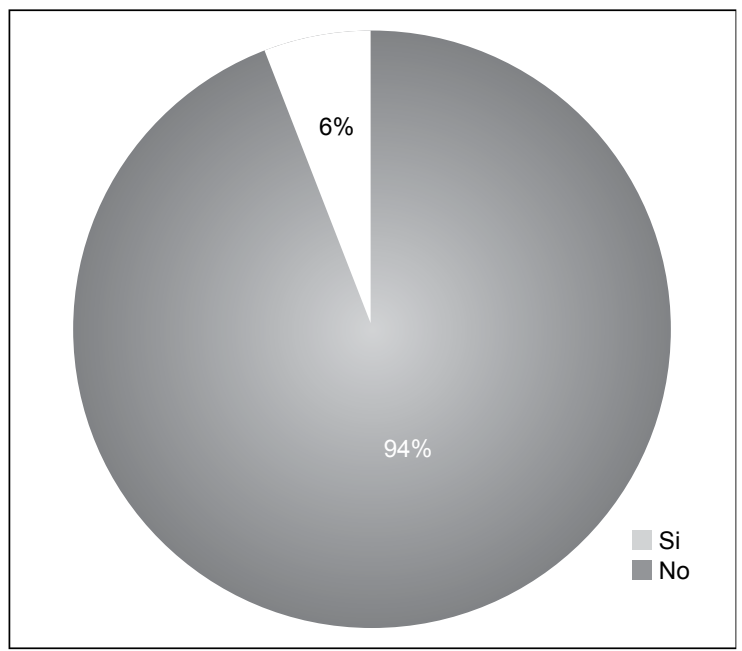

Fuente: elaboración propia.

El $94 \%$ de las empresas encuestadas realizan actividades de alto riesgo, de éstas el $44 \%$ han elaborado procedimientos documentados para trabajo seguro, y el $56 \%$ restante manifiesta no contar con dichos procedimientos.

Figura 3. Empresas que han establecido procedimientos de trabajo seguro.

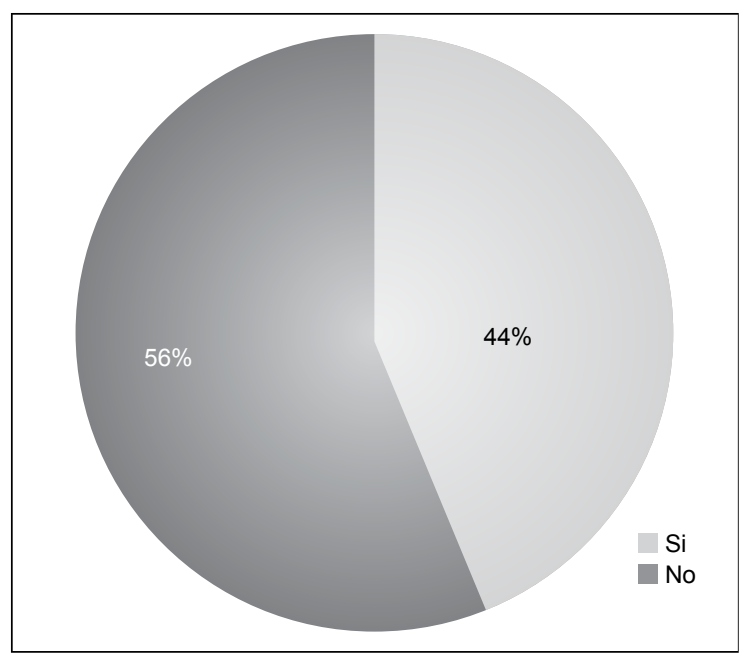

Fuente: elaboración propia.
Las encuestan permiten ver que el $76 \%$ de las empresas han tenido accidentes de trabajo (AT), mientras que el $24 \%$ manifiestan que no se han generado AT. Sin embargo, se encontró que el $65 \%$ de las empresas no realizan investigación sobre estos casos. Dentro de las causas de los accidentes de trabajo más comunes y su frecuencia, se encuentran:

\section{Cuadro 1. Causas de los accidentes} de trabajo más comunes.

\begin{tabular}{|c|c|}
\hline CATEGORÍA & PORCENTAJE \\
\hline Manipulación de equipo/herramienta & $76,0 \%$ \\
\hline Golpeado por equipos & $12,0 \%$ \\
\hline Colisión vehículos & $6,3 \%$ \\
\hline Caida a nivel & $0,0 \%$ \\
\hline Caida de altura & $6,3 \%$ \\
\hline Falta de entrenamiento & $12,0 \%$ \\
\hline
\end{tabular}

Fuente: elaboración propia.

El $47 \%$ de la población objeto de estudio realiza estadísticas de accidentes de trabajo, el restante $53 \%$ no realiza dichas estadísticas. Con relación a los exámenes médicos ocupacionales, se observó que el $65 \%$ de las empresas realiza exámenes médicos de ingreso, el $29 \%$ realiza exámenes médicos periódicos y el $53 \%$ realiza exámenes de retiro. Sin embargo, el $35 \%$ de las empresas encuestadas no cumplen con realizar exámenes médicos ocupacionales a sus trabajadores.

En una baja proporción, el $24 \%$ de la población cuenta con programas documentados de medicina preventiva, con las siguientes frecuencias: 
Cuadro 2. Causas de los accidentes de trabajo más comunes.

\begin{tabular}{|c|c|}
\hline CATEGORÍA & PORCENTAJE \\
\hline Farmacodependencia & $18,0 \%$ \\
\hline Manejo del riesgo psicosocial & $12,0 \%$ \\
\hline Nutrición & $0,0 \%$ \\
\hline Comportamiento autocuidado & $6,0 \%$ \\
\hline Educación sexual & $6,0 \%$ \\
\hline Estilos de vida saludables & $18,0 \%$ \\
\hline
\end{tabular}

Fuente: elaboración propia.

Con relación a la presencia de enfermedades laborales, el $100 \%$ de la población encuestada manifiesta que los trabajadores no han adquirido enfermedades de origen laboral. Sin embargo, el $100 \%$ de las empresas encuestadas no llevan a cabo estadísticas de enfermedad laboral.

El nivel de cumplimiento de la legislación en las diecisiete empresas estudiadas se presenta en la Figura 5.
Además de los resultados obtenidos a partir del cuestionario, se llevaron a cabo correlaciones entre los datos de las variables: por un lado se relacionó el cumplimiento de la legislación y la tasa de accidentalidad, y por otro lado, se relacionó la existencia de un sistema de gestión de seguridad y salud en el trabajo con la tasa de accidentalidad. Este análisis permite afirmar que existe una asociación entre las variables: por ejemplo, hay relación directa entre el cumplimiento de la legislación y el nivel de accidentalidad. Por otro lado, para las empresas objeto de estudio se observó que no se encuentra incidencia entre la existencia de un sistema de gestión de seguridad y salud en el trabajo sobre el nivel de accidentalidad.

\section{DISCUSIÓN}

Las empresas con mayores valores dentro del puntaje de cumplimento de legislación presentan un mayor número de accidentes de trabajo (AT). Este resultado

Figura 5. Nivel de cumplimiento de la legislación.

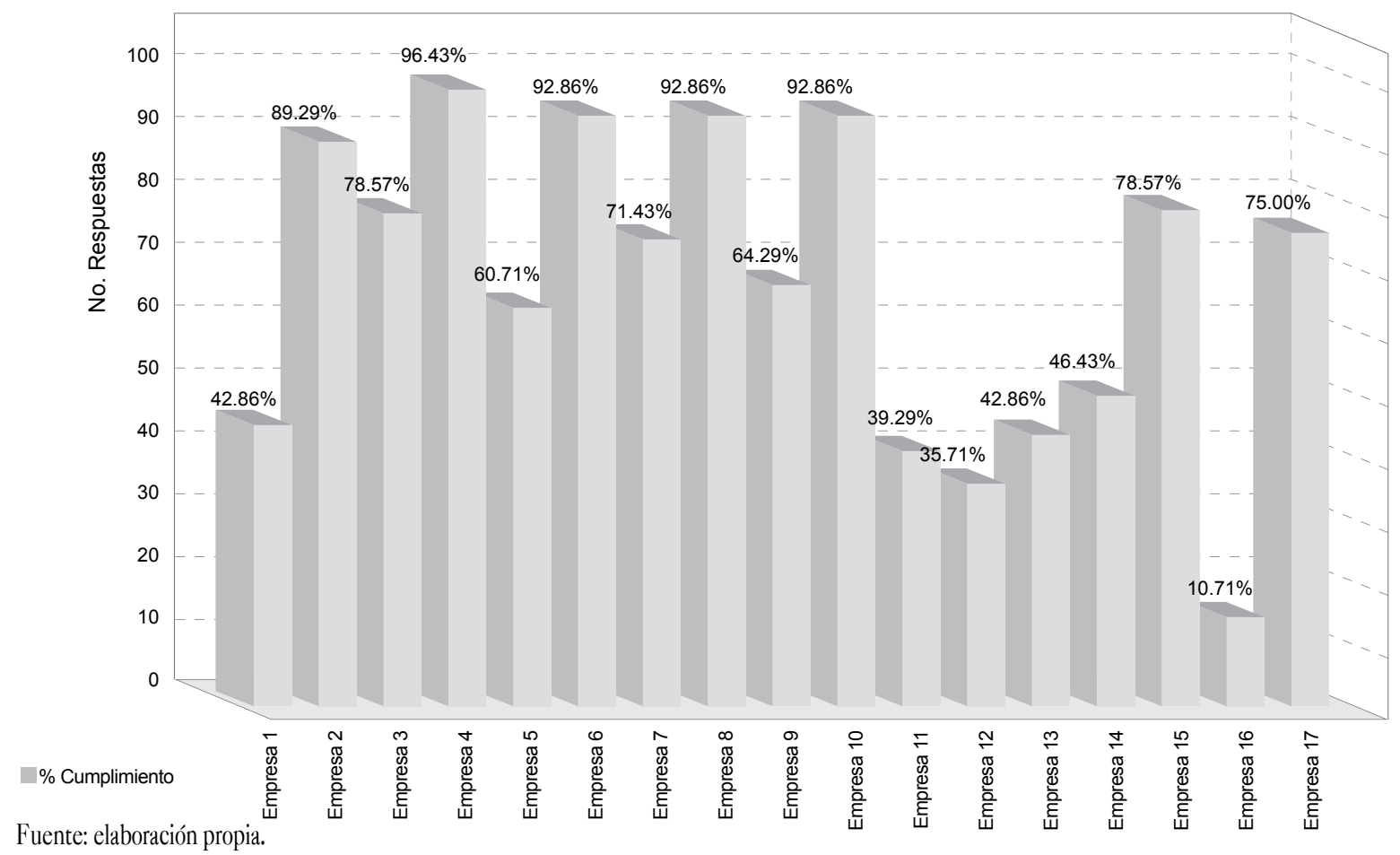


nos sorprende porque lo esperado sería que a mayor cumplimiento de la legislación, menor AT se presentarín. Este resultado se sustenta debido a que en la medida que se tenga conocimiento y cumplimiento de la legislación, mayor es el número de eventos registrados, cuantificados e investigados en las empresas que son catalogados como AT. Esto no sucede con las empresas que obtuvieron un nivel de cumplimiento medio y bajo. Como se puede observar, de las 9 empresas que se encuentran dentro del grupo de nivel de cumplimiento alto y que manifiestan haber tenido AT, 7 afirman que realizan estadísticas de AT. De las $7 \mathrm{em}$ presas que se encuentran dentro del grupo de nivel de cumplimiento medio, todas afirman que no investigan los accidentes e incidentes de trabajo, y 6 manifiestan que no realizan estadísticas de AT.

En relación al nivel de cumplimiento bajo manifiestan que no notifican los AT a la Administradora de Riesgos Laborales (ARL), no investigan las causas de los AT y no realizan estadísticas de AT. Como se observa en los resultados anteriores, el hecho de no mantener estadísticas de accidentalidad impide conocer el número real de accidentes laborales y deja este valor a la memoria del encuestado. Sin embargo, el no investigar los AT por parte de las empresas representa una debilidad en su gestión de seguridad y salud en el trabajo, ya que según la teoría de Hernández ${ }^{7}$ la investigación de accidentes permite afirmar que realizar una adecuada investigación de éstos es la única forma de estar en condiciones de tomar medidas que impidan su repetición.

A partir de los resultados de la investigación podemos afirmar que, para los casos seleccionados, la existencia de un SG-SST favorece y contribuye a la disminución

7 HERNANDEZ, Jacinto B. Investigación de Accidentes y Análisis de Fallas de Barreras Preventivas. En: Revista Científica Ingeniería Energética de la Unión Eléctrica de Cuba [en línea]. 2007, Vol. XXVIII, No. 1/2007. [citado en 2012-02-24]. 27 p. Disponible en Internet: http://rie.cujae.edu.cu/index.php/RIE/issue/view/12. de accidentes. Estos resultados van de la mano con lo mencionado por Health and Safety Executive (HSE) ${ }^{8}$ que manifiesta que la legislación por sí sola no es suficiente para garantizar la baja presencia de accidentes de trabajo y enfermedades laborales; así mismo, resalta la importancia de la implementación de SG-SST en aras de minimizar los índices de accidentalidad y fatalidad.

Las empresas encuestadas manifiestan que dentro de las causas con mayor frecuencia de AT los trabajadores son golpeados por equipos o por vehículos, lo cual coincide con las causas expuestas por National Institute For Occupational Safety And Health (NIOSH) 9 . Para prevenir este tipo de sucesos, NIOSH ${ }^{10}$ recomienda realizar diagramas detallados para ayudar a visualizar las áreas alrededor de los camiones de construcción y equipos diversos que no pueden verse en la posición del operador, junto con el uso de sistemas de alarmas como sensores, radares y sistemas de cámaras. Por otro lado, la falta de entrenamiento de los trabajadores también se suma a las causas de AT manifestadas por la población encuestada. Como medida de prevención, el autor Rubio recomienda que se debe incluir en la gestión de la seguridad y la salud en el trabajo actividades

8 HEALTH AND SAFETY EXECUTIVE. Revitalizing Health and Safety in Construction [en línea]. December, 2002. London. [citado en 2011-05-03]. p. 6-7. Disponible en: www.hse.gov.co.uk

9 NATIONAL INSTITUTE FOR OCCUPATIONAL SAFETY AND HEALTH. Building Safer Highway Work Zones: Measures to Prevent Workers Injuries from Vehicles and Equipment [online]. PRATT, Stephanie, FOSBROKE, David y MARSH, Suzanne. Publication No. 2001-128. Cincinnati. April 2001. [citado en 2011-05-03]. 5 p. Disponible en: http://www.cdc.gov/niosh/ docs/2001-128/pdfs/2001-128.pdf

10 NATIONAL INSTITUTE FOR OCCUPATIONAL SAFETY AND HEALTH. Evaluation of Systems to Monitor Blind Areas Behind Trucks Used in Road Construction and Maintenance: Phase 1 [en línea]. RUFF, Todd. Publication No. 2003-113. Pittsburgh, PA. February 2003. [consultado en 2011-05-03].15 p. Disponible en: http://www.cdc.gov/niosh/mining/pubs/pdfs/ri9660.pdf 
que permitan enseñar a los trabajadores tener un buen manejo de las señales de prevención en las obras ${ }^{11}$. Lo anterior va de la mano con que los AT no solo acarrean pérdidas humanas, sino también generan pérdidas financieras, lo que hace urgente mejorar las medidas de seguridad y salud ${ }^{12}$.

Se observa una tendencia en aquellas empresas que obtuvieron un nivel de cumplimiento bueno de la legislación a reportar un mayor número de accidentes de trabajo, en comparación con aquellas empresas que obtuvieron un nivel de cumplimiento medio y bajo de la legislación, las cuales en algunos casos reportaron cero accidentes de trabajo.

Lo anterior puede generarse debido a que el $53 \%$ de la población encuestada no mantienen estadísticas de accidentalidad. Dentro de las empresas con nivel medio de cumplimiento solo el $14 \%$ realiza estadísticas de accidentalidad y de la proporción de empresas con bajo nivel de cumplimiento no se mantienen estadísticas de accidentalidad.

Se observa una débil gestión de seguridad y salud en el trabajo implementada por las empresas encuestadas. Esto se evidencia en la falta de medidas preventivas, tales como: la elaboración de procedimientos documentados para trabajo seguro, plan de emergencia, programa de mantenimiento preventivo, realización de simulacros de emergencias, aplicación de inspecciones de seguridad, capacitaciones al personal en seguridad y salud en el trabajo y en atención a emergencias, entre otros, a través de las cuales se puedan detectar riesgos

11 RUBio ROMERO, Juan Carlos, et al. Manual de Coordinación de Seguridad y Salud en las Obras de Construcción. España: Díaz de Santos, 2005. 113 p.

12 TREGENZA, Tim. Building in safety. Actions to Improve Safety And Health in Construction. Magazine of the European Agency for Safety and Health at Work [online]. 2004. $n^{\circ} 7$. [consultado en 2011-05-03]. p. 16-17. Disponible en: http://osha.europa.eu/en/ publications/magazine/7. ISSN 1608-4144. y peligros e identificar medidas que conlleven a la prevención de accidentes y enfermedades profesionales. La prevención de accidentes y enfermedades es el objetivo que debería llevarse a cabo al interior de la organización a partir de una gestión en seguridad y salud en el trabajo.

\section{CONCLUSIONES}

La primera prueba de Chi cuadrado evidencia la falta de correlación entre el sistema de gestión de seguridad y salud en el trabajo sobre el nivel de accidentalidad dentro de la población objeto de estudio. Lo anterior puede ser el resultado de los bajos niveles de aplicación de los sistemas de gestión de seguridad y salud en el trabajo que tienen las empresas de la muestra.

La segunda prueba de Chi cuadrado permite afirmar que existe asociación entre las variables - i.e. cumplimiento de la legislación y el nivel de accidentalidad-, pero con una relación directamente proporcional, es decir, a mayor cumplimiento mayor accidentalidad. Esto sucede porque las empresas con bajos niveles de cumplimiento de la legislación no reportan con certeza los accidentes que se presentan.

Pese a que no se puede afirmar categóricamente que exista una relación entre la inexistencia de un sistema de seguridad y salud en el trabajo SG-SST y la presencia de accidentes en una organización, la proporción de empresas con accidentes que no cuentan con un sistema de seguridad y salud en el trabajo es 10 de 17. En este sentido, el $59 \%$ de estas empresas presentaron accidentes pero no cuentan con un sistema de seguridad y salud en el trabajo implementado. Así mismo, se observó en las empresas encuestadas que aquellas que cuentan con un SG-SST obtuvieron un mayor nivel de cumplimiento de la legislación, sobre aquellas empresas que no cuentan con dicho sistema de gestión. 
En cuanto al cumplimiento de los requisitos legales en materia de seguridad y salud en el trabajo que están obligadas a cumplir las empresas, se concluye que el $53 \%$ de la población encuestada obtuvo un resultado de cumplimiento alto, el $41 \%$ de la población obtuvo un resultado de cumplimiento medio y el $6 \%$ restante de la población obtuvo un resultado de cumplimiento bajo. En este sentido, la proporción de cumplimiento medio y bajo constituye un 47\%, que representa casi la mitad de la población, y la otra mitad, representado por el $53 \%$, tienen un nivel de cumplimiento bueno. El $11 \%$ de las empresas encuestadas ha implementado un sistema de gestión de seguridad y salud en el trabajo según el estándar RUC del Consejo Colombiano de Seguridad ${ }^{13}$. Ninguna de las otras empresas ha implementado un sistema de gestión de seguridad y salud en el trabajo según la norma OHSAS $18001^{14}$ u otro estándar relacionado.

La existencia de la legislación en seguridad y salud en el trabajo no es suficiente para la disminución de accidentes de trabajo y enfermedades laborales, siendo necesaria la implementación de un sistema de gestión de seguridad y salud ocupacional, dentro de los cuales se encuentra la norma NTC-OHSAS 18001.

Dentro de los sistemas de gestión en seguridad y salud ocupacional que existen en Colombia se encuentran el estándar NTC-OSHAS 18001 y el RUC del Consejo Colombiano de Seguridad. Sin embargo, a diferencia de Estados Unidos, no existe un estándar en seguridad y salud ocupacional relacionado a la construcción de obras viales. En Estados Unidos existe la norma ANSI/

13 CONSEjO COLOMBIanO DE SEGURIDAD. Guía del Sistema de Seguridad, Salud en el trabajo y Ambiente para Contratistas. OAUPE009. Rev. 18. Bogotá D.C.: El Consejo, 2013.

14 INSTITUTO COLOMBIANO DE NORMAS TÉCNICAS Y CERTIFICACIÓN. Sistemas de Gestión de Seguridad y Salud en el trabajo: Requisitos. NTC-OHSAS 18001. Bogotá D.C.: El Instituto, 2007. p. 2-11
ASSE A10.47 e igualmente existen los estándares FHWA, ASTM Y NCHRP 350, entre otros.

\section{REFERENCIAS}

AMERICAN NATIONAL STANDARDS INSTITUTE. Work Zone Safety for Highway Construction American National Standard for Construction and Demolition Operations. ANSI/ASSE A10.47. United States of America: American Society of Safety Engineers, 2009. 5 p.

CONSEJO COLOMBIANO DE SEGURIDAD. Guía del Sistema de Seguridad, Salud en el trabajo y Ambiente para Contratistas. OAUPE009. Rev. 18. Bogotá D.C.: El Consejo, 2013.

FEDERACIÓN DE ASEGURADORES COLOMBIANOS. Indicadores Técnicos: Encuesta Sistema de Información Gremial. Sistema de Información Estadística [en línea]. Bogotá, D.C. 2011. [Consultado en 2012-11-25]. Disponible en Internet: http://www. fasecolda.com/fasecolda/BancoConocimiento/R/ riesgos_profesionales_estadisticas_del_ramofinal_indicadores_tecnicos/riesgos_profesionales_-_estadisticas_del_ramofinal_-_indicadores_tecnicos.asp

FEDERACIÓN INTERAMERICANA DE LA INDUSTRIA DE LA CONSTRUCCIÓN. Evolución de la Economía de los Países Miembros de la FIIC 2009-2010. México, D.F. Octubre, 2010. [Consultado en 2012-07-12]. 42 p. Disponible en Internet: http://www.fiic.la/documentos/Evolucion\%20 Economia\%20Paises\%20FIIC\%202009-2010.pdf

HEALTH AND SAFETY EXECUTIVE. Revitalizing Health and Safety in Construction [en línea]. Diciembre, 2002. Londres. [consultado en 201205-03]. p. 6-7. Disponible en internet: www.hse. gov.co.uk 
HERNANDEZ, Jacinto B. Investigación de Accidentes y Análisis de Fallas de Barreras Preventivas. Revista Científica Ingeniería Energética de la Unión Eléctrica de Cuba [en línea]. 2007, Vol. XXVIII, No. 1/2007. 27 p. [consultado en 2012-02-24]. Disponible en Internet: http://rie.cujae.edu.cu/index. php/RIE/issue/view/12.

\section{INSTITUTO COLOMBIANO DE NORMAS TÉCNI-} CAS Y CERTIFICACIÓN. Sistemas de Gestión de Seguridad y Salud en el trabajo: Requisitos. NTC-OHSAS 18001. Bogotá D.C.: El Instituto, 2007. p. 2-11.

LI, Yingfeng y BAI, Yong. Comparison of characteristics between fatal and injury accidents in the highway construction zones. Safety Science [en línea]. 2008, vol. 46. 657 p. [Consultado en 2012-05-03]. Disponible en Internet: http://www.workzonesafety.org/files/documents/database_documents/ Publication8794.pdf. ISSN 0925-7535.

NATIONAL INSTITUTE FOR OCCUPATIONAL SAFETY AND HEALTH. Building Safer Highway Work Zones: Measures to Prevent Workers Injuries from Vehicles and Equipment [en línea]. PRATT, Stephanie, FOSBROKE, David y MARSH, Suzanne. No. 2001-128. Cincinnati: Abril 2001. 5 p. [Consultado el 2012-05-03]. Disponible en Internet: http://www. cdc.gov/niosh/docs/2001-128/pdfs/2001-128.pdf

\section{NATIONAL INSTITUTE FOR OCGUPATIONAL} SAFETY AND HEALTH. Evaluation of Systems to Monitor Blind Areas Behind Trucks Used in Road Construction and Maintenance: Phase 1 [en línea]. RUFF, Todd. No. 2003-113. Pittsburgh, PA. Febrero 2003. 15 p. [citado el 2012-05-03]. Disponible en Internet: http://www.cdc.gov/niosh/ mining/pubs/pdfs/ri9660.pdf
RUBiO ROMERO, Juan Carlos, et al. Manual de Coordinación de Seguridad y Salud en las Obras de Construcción. España: Díaz de Santos, 2005. 113 p.

THE CENTER FOR CONSTRUCTION RESEARCH AND TRAINING. Asthma in Heavy and Highway Construction Workers Exposed to Silica [en línea]. OLIVER, L. Christine y MIRACLE-MGMAHILL, Heidi. Brookline, Massachusetts: Julio 2003. p. 4-5 [citado el 2012-05-03]. Disponible en Internet: http://www.cpwr.com/pdfs/pubs/ research_pubs/oliver_Asthma.pdf

TREGENZA, Tim. Building in safety. Actions to Improve Safety And Health in Construction. En: Magazine of the European Agency for Safety and Health at Work [en línea]. 2004. Vol 7. p. 16-17 [citado el 2012-05-03]. Disponible en Internet: http://osha.europa.eu/en/publications/magazine/7. ISSN 1608-4144.

YUNI, José y URBANO, Ariel. Técnicas para investigar II: recursos metodológicos para la preparación de proyectos de investigación. $2^{\circ}$ ed. Córdoba (Argentina): Brujas, 2006. p. 15-29. ISBN 987-591-020-1. 